\title{
ONDE OS DIAMANTES OCORREM: PLANTAS INDICADORAS DE DIAMANTES NO BRASIL
}

\author{
WHERE THE DIAMONDS OCCUR: INDICATOR PLANTS OF DIAMOND GEMS IN BRAZIL
}

\author{
Bernardo TOMCHINSKY, Felipe Fernando da Silva SIQUEIRA
}

Universidade Federal do Sul e Sudeste do Pará, Instituto de Estudos em Saúde e Biológicas, Faculdade de Biologia. Folha 31, Quadra 07, Lote Especial, s/n Nova Marabá, Marabá, Pará, Brasil. CEP 68507-590. E-mail: btomchinsky@unifesspa.edu.br

\section{RESUMO}

As plantas indicadoras são espécies adaptadas a determinados ambientes que podem ser utilizadas pelas populações humanas para a classificação destas paisagens de acordo com as suas caraterísticas ou potenciais usos. Entre estas plantas, algumas são utilizadas como indicadoras geobotânicas por estarem relacionadas à presença de determinados minerais ou propriedades do solo. Este trabalho faz um levantamento de espécies consideradas indicadores de diamantes no Brasil. A partir de ampla revisão de literatura foram identificadas cinco espécies vegetais relacionadas com a ocorrência de diamantes no país (Babarcenia sp., Vellozia sp., Lageonocarpus adamantinus, Schwartzia adamantium e Norante guianensis). Destas, três foram analisadas quanto as suas distribuições e comparadas com os locais com registro de ocorrência de diamantes no país. Existe uma sobreposição entre as áreas onde estas plantas ocorrem e locais diamantíferos. É provável que as espécies de ocorrência mais restrita (L. adamantinus e S. adamantium) são melhores indicadoras ambientais para a ocorrência de diamantes. Entretanto, com os dados obtidos neste trabalho, apenas a ocorrência destas plantas não é suficiente como indicador da presença de diamantes e são necessários outros estudos para a prospecção geológica de corpos kimberlíticos e de gemas de diamantes em fontes secundárias onde estas espécies ocorrem para confirmar esta correlação.

PALAVRAS-CHAVE: etnoecologia, etnopedologia, indicador geobotânico, conhecimento tradicional

\begin{abstract}
Indicator plants are species adapted to determinate environments that can be used by human populations to classify these environments. Among these plants, some are used as geobotanical indicators and are related to the presence of certain minerals or soil properties. This work is a survey of species considered indicators of diamond gems in Brazil. From literature review, five species of plants related to the occurrence of diamonds in Brazil were identified (Babarcenia sp., Vellozia sp., Lageonocarpus adamantinus, Schwartzia adamantium e Norante guianensis). Of three of these species their distribution were analyzed and compared with diamond areas in Brazil. We found an overlap between the areas where these plants grown and diamond sites. We believe that more restricted species (L.adamantinus $e$ S. adamantium) are better environmental indicator for the occurrence of diamond. However, with the data used in this work, we can't say that just the occurrence of these plants is sufficient as an indicator of the presence of diamonds. Further field studies are needed with geological prospecting of kimberlite bodies and diamond gems in secondary sources where these plants occur to confirm this correlation.
\end{abstract}

KEYWORDS: ethnoecology, ethnopedology, geobotany indicator, traditional knowledge

\section{INTRODUÇÃO}

As espécies vegetais evoluíram nos mais diversos tipos de ambiente, adaptando-se às características locais (LEIMU e FISCHER, 2008). Altitude, características do solo (como textura, disponibilidade hídrica, nutrientes e $\mathrm{pH}$ ), atmosfera, pluviosidade, umidade relativa do ar, temperatura, polinizadores, dispersores, luminosidade e fotoperíodo podem ser alguns dos fatores limitantes ao desenvolvimento dos vegetais nestes diferentes ambientes (GUREVITICH et al., 2009). Modelos biogeográficos tentam explicar a distribuição de cada espécie em função destas diversas variáveis. Desta forma, a ocorrência de determinadas espécies pode estar associada a algumas destas características limitantes, fazendo destas plantas potenciais indicadoras da paisagem. Cabe, entretanto, ao ser humano aprender a observar e identificar tais espécies bioindicadoras de acordo com suas necessidades e especificidades. 
Indicadores geobotânicos são plantas com ocorrência limitada, associada ou com diagnose foliar visual de toxidez ou deficiência de nutrientes, relacionadas a condições específicas das propriedades geológicas dos ecossistemas, como a presença e acúmulo de determinados elementos no solo, distribuição de rochas no terreno, profundidade e perfil do solo, presença de corpos de água subterrâneos, áreas halófitas, depósitos minerais, entre outros (MARTIN e GOUGHTREY, 1982; ALLABY, 2020).

Desde o século XVII, a espécie Silene suecica (Lodd.) Greuter \& Burdet. (Caryophyllaceae), conhecida como planta-pirita, é associada à ocorrência de minas de cobre na Escandinávia, o que atualmente compreende-se que ocorre devido a sua tolerância a níveis tóxicos neste minério (NORDAL et al., 1999). Na África, Ocimum centraliafricanum R.E.Fr., conhecida localmente como copper plant, também é associada a áreas com cobre (BROOKS, 1992). Cannon (1971) lista 122 espécies indicadoras de reservas de minério (indicadoras geobotânicas) em todo o mundo e Brooks (1979) analisa 85 espécies plantas consideradas indicadoras de diversos minerais ( $\mathrm{Al}, \mathrm{Co}, \mathrm{Cu}, \mathrm{Au}, \mathrm{Fe}, \mathrm{Mg}, \mathrm{Ni}, \mathrm{Bo}, \mathrm{Se}, \mathrm{Ag}, \mathrm{U}, \mathrm{Zn}$ ), e observa que, entre elas, as famílias Caryophyllaceae, Fabaceae e Lamiaceae são as mais frequentes.

No Brasil, é comum o uso de plantas ruderais como indicadoras da fertilidade do solo por agricultores (LORENZI, 2008; PRIMAVESI, 2017). Entre os povos indígenas, Posey (1986) descreve como os Kayapó classificam os diferentes tipos de ambientes que ocupam a partir da ocorrência de diferentes espécies de plantas indicadoras de paisagens.

Recentemente, Haggerty (2015) comprovou que a espécie Pandanus candelabrum P. Beauv (Pandanaceae) ocorre exclusivamente em áreas com afloramento de diamantes na África Ocidental (Libéria), conforme o conhecimento tradicional previa. O autor descobriu que sua ocorrência é restrita às localidades com afloramento de chaminés de kimberlito, uma formação geológica proveniente de rochas magmáticas sob alta temperatura e pressão e que ocasionalmente forma diamantes (JEANSEN e SHEAHAN, 1995; NIXON, 1995; MITCHELL, 2008). Estes kimberlitos podem ser ricos em diferentes minerais, como mica, calcita, diopsídio e apatita (BULANOVA, 1995; GRIFFIN e RYAN, 1995), o que pode gerar um solo de composição e fertilidade especifica contribuindo para o estabelecimento de uma vegetação adaptada a estas condições ambientais.

A associação entre P. candelabrum e as chaminés de kimberlito foi relacionada da seguinte forma: devido às formações tubulares encontradas no kimberlito ocorre um aumento da capacidade de retenção d'água, tornando-o mais argiloso do que os horizontes adjacentes; as raízes de $P$. candelabrum penetram nessas chaminés para se fixar; durante o crescimento de $P$. candelabrum as raízes absorvem seletivamente íons de $\mathrm{Mg}, \mathrm{K}, \mathrm{P}$ e diminuem a concentração de Fe; e durante o crescimento das raízes desta espécie ocorre a elevação de rochas ígneas (e.g. ilmenita: mineral de magnetismo fraco $\left[\mathrm{FetiO}_{3}\right]$ ) e eventualmente pequenos diamantes (KLAPPA, 1980; HAGGERTY, 2015). Esta associação está auxiliando os governos dos países da África Ocidental na localização de depósitos de diamantes (HAND, 2015).

No Brasil, país com áreas de exploração de diamante conhecidas desde o século XVIII associado a uma atividade de mineração artesanal, tem-se registrado relatos sobre espécies vegetais relacionadas à ocorrência desta pedra preciosa (SPIX e MARTIUS, 1824; CORREA, 1984; SILVA JUNIOR, 2005; PECKOLT e PECKOLT, 2007). Entretanto, a falta de um levantamento sistemático das espécies vegetais relacionadas à ocorrência de diamantes no Brasil dificulta a produção de estudos que procurem confirmar esta correlação.

Desta forma, este trabalho tem como objetivo realizar um levantamento das espécies de plantas relatadas como indicadoras de diamantes no Brasil a fim de fornecer subsídios para futuros estudos que relacionem as possíveis espécies candidatas com variáveis geológicas.

\section{MATERIAL E MÉTODOS}

As espécies vegetais indicadoras de diamantes no Brasil foram levantadas a partir de extensa revisão de literatura em livros, periódicos e teses. Foram utilizadas as seguintes bases de dados de literatura científica: Scopus (https://www.scopus.com), Google Scholar (http://scholar.google.com.br), Scielo: (http://www.scielo.org), Periódicos da capes (http://www.periodicos.capes.gov.br) para a combinação das palavras "diamantes", "Brasil", "plantas bioindicadoras", "plantas geobotânica" e "plantas indicadoras", sem limitação por ano por ano de publicação.

As espécies identificadas tiveram o seu nome atualizado de acordo com a APG IV na Flora do Brasil 2020 (2020) e trópicos (2019) e foram realizadas buscas especificas nas bases de dados já citadas com o nome válido e sinônimos para identificação de outros usos e descrição morfológica. 
A distribuição e áreas de ocorrência destas espécies foram conferidas a partir das bases de dados do Herbário Virtual (INCT, 2020), CNCFlora (2018, 2020), trópicos (2019) e Flora do Brasil 2020 (2020) e comparado com regiões de ocorrência de diamantes no Brasil, a partir dos mapas e documentos elaborados pelo Serviço Geológico do Brasil (CPRM) e de outras fontes consultadas.

\section{RESULTADOS}

\section{Ocorrência de diamantes no Brasil}

O Brasil possui registros da exploração de diamantes desde o início do século XVIII, quando foram descobertos depósitos na região de Diamantina no estado de Minas Gerais. Até o século XIX o país figurava entre os maiores produtores mundiais, quando foram descobertas jazidas na África do Sul (SVISERO e CHIEREGATI, 1991; VALE, 2003; COSTA e LUZ, 2005). Ao longo dos séculos foram encontrados depósitos de diamante em diversas regiões do Brasil, desde o Sul do país em Tibaji, no estado do Paraná; Itararé e Patrocínio Paulista em São Paulo; Alto Parnaíba e região Central de Minas Gerais; Aragarças e Piranhas em Goiás; Barra dos Garças, Chapada dos Guimarães, Aripuanã e Juína no Mato Grosso; Coxim no Mato Grosso do Sul; Chapada Diamantina na Bahia; Gilbués no Piauí; Imperatriz no Maranhão; Marabá no Pará; Oeste de Rondônia, além de localidades menores no Amapá e Roraima; com destaque para os estados de Minas Gerais, com $71 \%$ das reservas oficiais Brasileiras, e Mato Grosso com 85\% da produção nacional (SVISERO e CHIEREGATI, 1991; VALE, 2003; LIMA, 2016; CABRAL NETO et al., 2017; NANNINI et al., 2017) (Figura 1). O projeto Diamantes do Brasil, do Serviço Geológico do Brasil (CPRM), concluído em 2017, mapeou 804 ocorrências de diamantes, 142 garimpos, 42 campos e 1.344 corpos kimberlíticos no país (SILVEIRA et al., 2018).

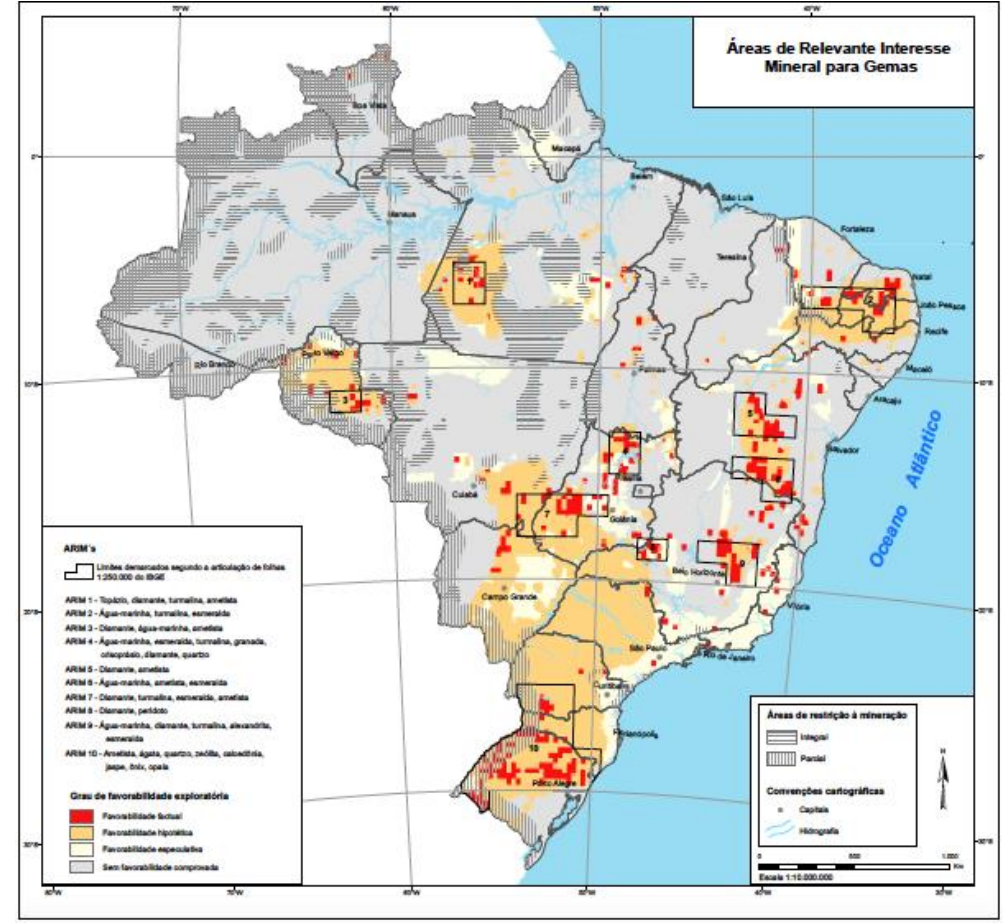

Figura 1 - mapa de ocorrência de diamantes no Brasil (Fonte: SILVEIRA et al., 2018).

\section{Espécies vegetais indicadores de diamante no Brasil}

Segundo a literatura consultada, foram identificadas cinco espécies vegetais relacionadas pelas populações locais com áreas de ocorrência de diamantes no Brasil, duas pertencentes à família Velloziaceae, uma pertencente à família Cyperaceae (Lagenocarpus adamantinus) e duas à família Marcgraviaceae (Schwartzia adamantium e Norantea guianensis). As duas espécies de Velloziaceae (Babarcenia Vand. e Vellozia Vand.), foram mencionadas por Spix e Martius (1824) e não foram analisadas quanto a sua distribuição, já que os autores não determinaram as espécies e há pelo menos 89 espécies destes gêneros que ocorrem na região de Diamantina descrita pelos autores (INCT, 2020). 
Lagenocarpus adamantinus Nees (Cyperaceae)

Nome popular: capim-dos-diamantes, brilhante, capim-brilhante, capim-diamante, tiririca-dosdiamantes.

Descrição e ocorrência: Erva terrícola ou rupícola, perene, de $70 \mathrm{~cm}$ a $1,1 \mathrm{~m}$ de altura, formando pequenas touceiras laxas, endêmica de campos rupestres do Cerrado no estado de Minas Gerais, com ocorrências registradas apenas na região de Diamantina e na Serra do Cipó (Figuras 2a e 2b). Ocorre sobre afloramentos quártzicos como colonizadora primária (ALVES et al, 2009). Por ocupar uma área restrita, é classificada como Vulnerável (VU) (FLORA DO BRASIL, 2020; CNCFlora, 2018). É crença popular que vegeta de preferência nos terrenos diamantíferos do estado de Minas Gerais na região de Diamantina (CORREA, 1982; PECKOLT e PEKCOLT, 2006).
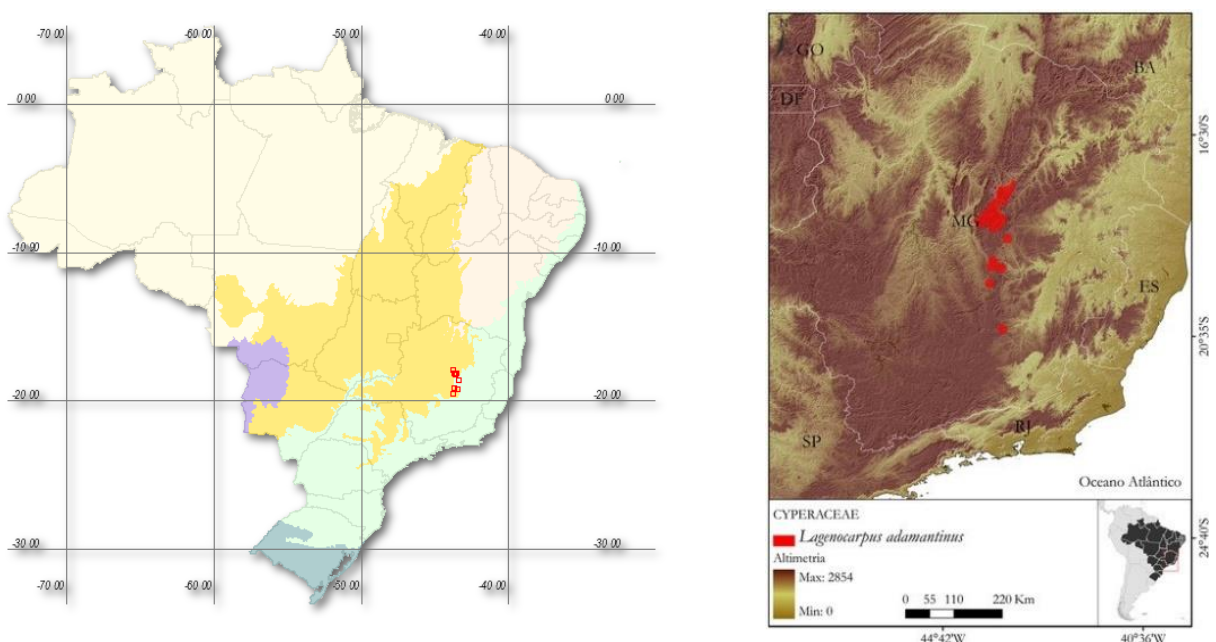

Figura 2a (esquerda): Coletas de Lagenocarpus adamantinus Nees (Cyperaceae) no Brasil. Fonte: (INCT, 2020).

Figura 2b (direita): Ocorrência e distribuição de Lagenocarpus adamantinus Nees (Cyperaceae) no Brasil (CNCFlora, 2018).

Schwartzia adamantium (Cambess.) Bedell ex Gir.-Cañas (Marcgraviaceae).

Nome local: agarra-pé, mel-de-arara, pau-de-papagaio.

Descrição e ocorrência: É um arbusto ou pequena árvore de até quatro metros de altura, caule tortuoso, geralmente com raízes adventícias, subcaducifólia ou caducifólia, terrícola, folhas simples, alternoespiraladas, subsésseis e glabras, nativa do Brasil e endêmica do Cerrado, com ocorrência em vegetação de campos rupestres, cerrado (Strictu sensu) e florestas ciliares em terrenos rochosos a mais de 900 metros de altitude (FLORA DO BRASIL, 2020, 2020). Possui 212 registros de coletas no Brasil (Figura 3) (INCT, 2020). Silva Junior (2005) relata que esta é uma espécie considerada indicadora de áreas com diamante na Chapada dos Veadeiros. Através de sua dispersão (Figura 3), é possível observar que ela ocorre em municípios da região da Chapada Diamantina, no estado da Bahia, Cristalina, Pirenópolis e Alto Paraíso, em Goiás e Diamantina em Minas Gerais, locais onde historicamente já foi realizada exploração de diamantes e cristais.

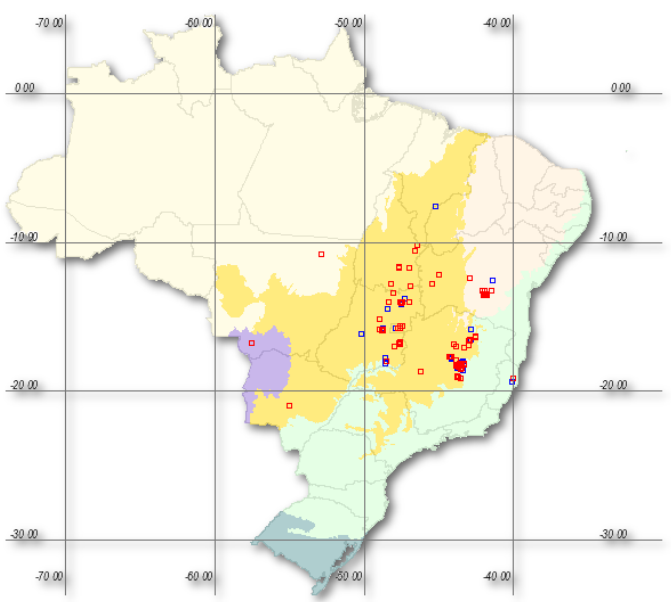

Figura 3: Ocorrência e distribuição de Schwartzia adamantium (Cambess.) Bedell ex Gir.-Cañas (Marcgraviaceae) no Brasil (INCT, 2020). 
Norantea guianensis Aubl. (Marcgraviaceae)

Nome local: flor-de-papagaio, planta-dos-diamantes, parreira-da-pedra, rabo-de-arara.

Descrição e ocorrência: Arbustiva volúvel com ramos horizontais a decumbentes, emitindo raízes avermelhadas ao longo do caule, hemiepífita, folhas obovais com base cuneada e ápice retuso, inflorescência em racemos terminais multiflorais com ocorrência desde o Suriname até o Brasil Central e Bolívia, com maior frequência na região amazônica, além de Caatinga e Cerrado em vegetação de campos rupestres, floresta ciliar, floresta de terra firme, savana amazônica e áreas de canga aberta (afloramento ferrífero) (TROPICOS, 2020; FLORA DO BRASIL, 2020, 2020). É uma espécie com ampla distribuição no Brasil e 446 coletas registradas (Figura 4) (INCT, 2020).

Correa (1982) e Silva Junior (2005) dizem que ela vegeta em terras áridas, às vezes diamantíferas, e por isso, o povo considera-a indicadora, sobretudo na Chapada dos Veadeiros. Entretanto, observamos que sua distribuição é muito mais ampla do que as áreas diamantíferas, ao comparar com as áreas com registro de ocorrência de diamantes no Brasil (Fig. 1).

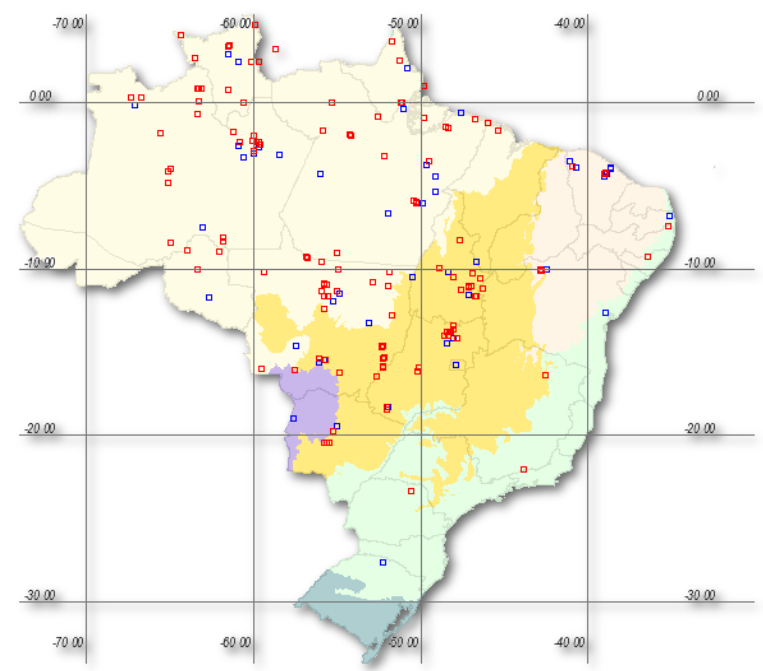

Figura 4: Ocorrência e distribuição de Norantea guianensis Aubl. (Marcgraviaceae) no Brasil (INCT, 2020).

\section{DISCUSSÃO}

Nenhumas das três espécies estudadas haviam sido citadas nos trabalhos mais completos relacionados anteriormente (CANNON, 1971; BROOKS, 1979) e tampouco pertencem às famílias mais frequentes (Lamiaceae, Fabaceae, Caryophyllaceae) apontadas por Brooks (1979) como geoindicadoras. As Velloziaceae (Barbacenia sp. e Vellozia sp.) relatadas por Spix e Martius (1824) também não haviam sido consideradas nestes trabalhos.

Os nomes científicos (válidos e sinônimos) das espécies podem carregar diversos significados atribuídos por seus autores. L. adamantinus e S. adamantium têm epíteto específico referente a diamante, que poderia ser relacionado tanto a região onde são encontradas (Diamantina na Serra do Espinhaço, estado de Minas Gerais) ou a sua relação com a ocorrência de diamante. Nas obras de referência onde foram publicadas as descrições destas espécies é feita relação apenas à região de ocorrência (SAINT HILAIRE, 1825; MARTIUS, 1842). Em pesquisa na Flora do Brasil 2020 (2020), é possível encontrar outras espécies vegetais nativas do Brasil com epíteto semelhante (Camponanesia adamantium, Croton adamantinus, Hyptis adamantium, Koanophyllon adamantium, Lavoisiera adamantium, Lepidaploa adamantium e Senecio adamantinus), todas com coletas significativas na região de Diamantina, sendo que destas $C$. adamantinus e $S$. adamantinus possuem apenas um registro de coleta cada, ambos na região de Diamantina (INCT, 2020).

Enquanto L. adamantinus tem distribuição mais restrita, ocorrendo apenas em uma pequena extensão de área onda há registro de ocorrência de diamantes, S. adamantium tem distribuição mais ampla, mas também sobreposta a regiões diamantíferas. Norantea guianesnsis, entretanto, tem a distribuição mais ampla, com registros em outras regiões do país não diamantíferas (INCT, 2020; TROPICOS, 2020). A distribuição mais restrita de algumas espécies pode ser um bom indicativo daquelas com maior potencial de indicador geobotânico, uma vez que essa distribuição restrita pode estar relacionada com variáveis geológicas locais. 
Morfologicamente, nenhuma das três espécies possui raízes escoras ou superficiais como $P$. candelabrum, que Haggerty (2015) relaciona com o soerguimento de gemas de diamante da superfície do solo.

Além dos fatores ambientais e ecológicos, outras questões podem estar envolvidas com a distribuição destas plantas, como o seu manejo e outras atividades antrópicas conforme sugerem Levis et al. (2017) para espécies arbóreas hiperdominantes na região amazônica, e Baléé $(1989,2013)$ para formações florestais amazônicas. Entretanto, não foram encontrados registros de uso por populações humanas para as três espécies estudadas nas referências consultadas, ainda que as plantas da família Marckgravariaceae (N. guianensis e S. adamatinum) tenham grande potencial ornamental, podendo, por isso, eventualmente ser cultivadas ou manejadas de alguma forma.

É interessante observar que no Brasil a exploração de diamantes foi feita historicamente de forma artesanal em fontes secundárias, na superfície do solo ou em aluvião (mineração em rios) (VALE, 2003; COSTA e LUZ, 2005), de modo que a maior parte das pedras garimpadas é proveniente de rochas desgastadas e podem ter sido arrastadas por longa distância da rocha matriz (MARSHALL e BAXTERBROWN, 1995) e, desta forma, o relato de garimpo de diamantes em determinada região no Brasil pode não ser necessariamente um indicativo da existência de chaminés de kimberlito e outros minerais associados na área.

É possível que outros indicadores geológicos sejam utilizados por garimpeiros para a identificação de áreas com diamantes como a ocorrência de cascalhos diamantíferos (CABRAL NETO et al., 2017). Esta hipótese pode ser válida e deve ser testada em outros estudos, pois no garimpo se dedica a maior parte do tempo analisando justamente as rochas. Em vídeos disponíveis na Internet são apresentados diferentes tipos de cascalho associados à presença de gemas, usualmente com nomes relacionados a sua forma, como feijão, figo/fígado-de-galinha, chiclete mascado e amendoim, ou a sua composição como quartzo, ferragem, cristal e hematita. A etnopedologia, área das etnociências que estuda a relação entre sociedades e solos (ALVES, 2005; ARAÚJO et al., 2013), pode contribuir com este trabalho a partir da identificação dos diferentes tipos de solo e rochas e seus potenciais de uso pelas populações locais.

Haggerty (2015) observou in loco o adensamento de P. candelabrum em áreas sobre chaminés de kimberlito já prospectadas e conhecidas na Libéria, o que não pode ser feito neste estudo já que os mapas de distribuição das plantas neste trabalho foram realizados a partir de coletas conhecidas destas espécies, sem a confirmação de ocorrência de kimberlitos nestes mesmos locais.

Apesar da comparação realizada entre as áreas de ocorrência das plantas estudadas e das áreas com registro de diamantes no Brasil, este estudo possui limitações quanto à precisão dos dados geográficos utilizados. O garimpo de diamantes ocorre em locais restritos e não em toda a extensão dos municípios listados pelo Serviço Geológico do Brasil (CPRM), e pela escala do mapa disponibilizado e utilizado (SILVEIRA et al., 2018) é impossível precisar os pontos exatos onde foram realizados estes registros de diamantes para aproximar dos locais onde as plantas foram coletadas, sendo que as chaminés de kimberlito possuem no máximo poucas centenas de largura (NIXON, 1995). Ao mesmo tempo não são todas as plantas coletadas, registradas e utilizadas neste trabalho que possuem localização precisa de sua ocorrência, podendo ter sua localização ajustada para a sede do município na ausência desta informação (INCT, 2020), dificultando a elaboração de mapas mais precisos. Também é necessário reconhecer que o mapa do CPRM utilizado indica apenas os locais onde já há a ocorrência de diamantes confirmada e não outros locais onde também poderiam ocorrer (SILVEIRA et al., 2018).

Para o aprimoramento da discussão deste trabalho seria interessante entrevistar garimpeiros na região de ocorrência destas plantas e em locais tradicionais de garimpo. Entretanto, tanto a caracterização da atividade do garimpo artesanal como ilegal quanto à discrição do garimpeiro com o local do garimpo ou das técnicas desenvolvidas para evitar possíveis concorrentes, dificultariam esta abordagem.

\section{CONCLUSÕES}

Foram encontradas cinco espécies vegetais nativas do Brasil relacionadas à ocorrência de diamantes no país nas referências consultadas, das quais apenas três foram identificadas até espécie. Apesar da distribuição destas três plantas sobreporem áreas diamantíferas, não é possível afirmar que apenas sua ocorrência é suficiente como indicadora de áreas diamantíferas e seriam necessários estudos de campo para confirmar esta correlação. É provável que as espécies de distribuição mais restrita sejam melhores indicadoras geobotânicas do que aquelas de ampla distribuição. Trabalhos de biogeografia a partir de um maior número de amostras coletadas, mais variáveis ambientais analisadas, associada a estudos de prospecção de minerais no Brasil devem contribuir no futuro para esta discussão. 


\section{AGRADECIMENTOS}

Agradecemos aos revisores que leram este manuscrito e fizeram valiosas sugestões para a sua melhora.

\section{REFERÊNCIAS}

ALLABY, M. Geobotanical exploration: a dictionary of ecology. Encyclopedia. 2020. Disponível em: <https://www.encyclopedia.com>. Acessado em: 04 jan 2020.

ALVES, M; ARAÚJO, A. C. VITTA, F. Cyperaceae. In: Giulietti, A. M. et al. (Org.). Plantas Raras do Brasil. Belo Horizonte: Conservação Internacional; Universidade Estadual de Feira de Santana. 2009. 496p.

ALVES, A. G. C. Conhecimento local e uso do solo: uma abordagem etnopedológica. Interciência, v. 30, n. 9, p: 524-528. 2005.

ARAÚJO, A.L., ALVES, A.G.C. ROMERO, E., FERREIRA, T.O. etnopedologia: uma abordagem das etnociências sobre as relações entre as sociedades e os solos. Ciência rural, v. 43, n.5, p: 854-860. 2013

BALÉE, W. Cultura na vegetação da Amazônia brasileira. In: NEVES, W. A. (Org.). Biologia e ecologia humana na Amazônia: Avaliação e perspectivas. Belém: SCT/PR, CNPq. 1989. p. 95-109.

BALÉE, W. Cultural forests of the Amazona: A history of people and their landscape. University of Alabama. 2013. 268 p.

BROOKS, R. R. Indicator plants for mineral prospecting - a critique. Journal of Geochemical Exploration. v. 12, p: 67-78. 1979. doi:10.1016/0375-6742(79)90064-5

BROOKS, R. R. Noble Metals and Biological Systems: Their Role in Medicine, Mineral Exploration, and the Environment. CRC Press. 1992. p. 181.

BULANOVA, G. P. The formation of diamond. Journal of Geochemical Exploration, v. 53, n: 1-3, p: 1-23. 1995. https://doi.org/10.1016/0375-6742(94)00016-5

CABRAL NETO, I., NANNINI, F., SILVEIRA, F. V., CUNHA, L. M. Áreas kimberlíticas e diamantíferas do estado de Minas Gerais e regiões adjacentes. Projeto Diamante Brasil. Informe de recursos minerais. Programa geologia do Brasil. Série pedras preciosas n ${ }^{\circ}$ 10. Brasília: CPRM. 2017.

CANNON, H. L. Use of plant indicators in ground water surveys, geologic mapping, and mineral prospecting. Taxon, v. 20, p: 227-256. 1971.

CNCFLORA. Lagenocarpus adamantinus in Lista Vermelha da flora brasileira versão 2012.2 Centro Nacional de Conservação da Flora. 2018. Disponível em http://cncflora.jbrj.gov.br/portal/ptbr/profile/Lagenocarpus adamantinus. (Acesso em 7 novembro 2018).

CNCFLORA. Koanophyllon adamantium in Lista Vermelha da flora brasileira versão 2012.2 Centro Nacional de Conservação da Flora. 2020. Disponível em <http://cncflora.jbrj.gov.br/portal/ptbr/profile/Koanophyllon adamantium>. (Acesso em 5 abril 2020).

CORREA, P. M. Dicionário das plantas úteis do Brasil e das exóticas cultivadas. Rio de Janeiro: IBDF, 6v. 1982.

COSTA, M.J., LUZ, A.B. DIAMANTE. IN. LUZ, A. B., LINS, F. A. F. Rochas e Minerais Industriais. Rio de Janeiro: CETEM/MCT: 427-450. 2005.

FLORA DO BRASIL 2020. Jardim Botânico do Rio de Janeiro. 2020. Disponível em http://floradobrasil.jbrj.gov.br (Acesso em 13 de fevereiro de 2020).

GRIFFIN, W. L.; RYAN, C.G. Trace elements in indicator minerals: area selection and target evaluation in diamond explorations. Journal of Geochemical Exploration, v. 52, n. 1-3, p: 311-337. 1995. https://doi.org/10.1016/0375-6742(94)00015-4

GUVERITCH, J., SCHEINER, S. M. S, FOR, G. A., OLIVEIRA, P. L., HARTZ, S. M., DUARTE, L. S., BECKER, F. G., DILLENBURG, L. R., MULLER, S. C. Ecologia vegetal. Porto Alegre: Artmed. 2009. 572 p.

HAGGERTY, S. E. Discovery of a kimberlite pipe and recognition of a diagnostic botanical indicator in Liberia. Economic Geology, v. 110, n. 4, p: 851-856. 2015. doi:10.2113/econgeo.110.4.851

HAND, R. Rare African plant signals diamonds beneath the soil. Sciencemag. 2015. Disponível em: https://www.sciencemag.org/news/2015/05/rare-african-plant-signals-diamonds-beneath-soil\# (Acesso em 27 de maio de 2020).

INCT. Herbário Virtual da Flora e dos Fungos. Instituto Nacional de Ciência e Tecnologia. 2020. Disponível em http://inct.splink.org.br, (acesso em 04 de Abril de 2020).

JEANSEN, A. J. A., SHEAHAN, P. A. Catalogue of world wide diamond and kimberlite occurrences: a selective and annotative approach. Journal of geochemical exploration. v. 53, n.1-3, p: 73-111. 1995. https://doi.org/10.1016/0375-6742(94)00017-6

KLAPPA, C. F. Rhizoliths in terrestrial carbonates: Classification, recognition, genesis and significance. Sedimentology, v. 27, n.6, p: 613-629. 1980.

LEIMU, R., FISCHER, M. A Meta-Analysis of Local Adaptation in Plants. PlosOne, v.3, n.12, p: e4010. 2008. https://doi.org/10.1371/journal.pone.000401

LEVIS, C. et al. Persistent effects of pre-Columbian domesticatication Amazonian forest composition. Science, v. 355, n. 6328, p: 925-931. 2017. DOI: 10.1126/science.aal0157 
LIMA, E. S. Aluviões diamantíferos da foz dos rios Jequitinhoa e pardo: fase I: projetos diamante Brasil: estado da Bahia. Informe de recursos Minerais, série pedras preciosas n. 9. Salvador: CPRM. 2016.

LORENZI, H. Plantas daninhas do Brasil: terrestres, aquáticas, parasitas e tóxicas. Nova Odessa: Instituto Plantarum. 2008. 640p.

MARSHALL, T. R., BAXTER-BROWN, R. Basic principles of alluvial diamond exploration. Journal of Geochemical Exploration, v. 53, n. 1-3, p: 277-292. 1995. https://doi.org/10.1016/0375-6742(94)00067-L

MARTIN, M. H., COUGHTREY, P. J. Biological Indicators of Natural Ore-Bodies: Geobotanical and Biogeochemical Prospecting for Heavy Metal Deposits. In: Biological Monitoring of Heavy Metal Pollution. Pollution Monitoring Series. Springer, Dordrecht. 1982.

MARTIUS, C.F.P.V. Lageonocarpus adamantinus. Flora brasiliensis. v.2, n. 3, p: 165. 1842.

MITCHELL, R.H. Petrology of hypabyssal kimberlites: Relevance to primary magma compositions. Journal

of Volcanology and Geothermal Research. v. 174, n: 1-3, p $1-8.2008 . \quad$ d oi.org/10.1016/j.jvolgeores.2007.12.024.

MOTA, N.F.O.; WATANABE, M,T.K.; ZAPPI, D.C.; HIURA, A.L.; PALLOS, J.; VIVEROS, R.S.;

GIULIETTI, A.M.; VIANA, P.L. Cangas da Amazônia: a vegetação única de Carajás evidenciada pela lista de fanerógamas. Rodriguésia, v. 69, n.3, p: 1435-1488. 2018. https://doi.org/10.1590/2175-7860201869336

NANNINI, F.; CABRAL NETO, I., SILVEIRA, F. V., CUNHA, L. M., OLIVEIRA, R. G., WESKA, R. K. Áreas kimberlíticas e diamantíferas do estado do Mato Grosso. Programa Diamante Brasil. Informe de recursos mineiras. Programa geologia do Brasil. Série Pedras Preciosas, nº 12. Brasilia: CPRM. 2017.

NIXON, P. H. The morphology and nature of primary diamontiferous occurrences. Journal of Geochemical

Exploration v. 53, n 1-3, p: 41-71. 1995. https://doi.org/10.1016/0375-6742(94)00034-9

NORDAL, I., HARALDSEN, K. B., ERGON, ̊. Copper resistance and genetic diversity in Lychnis alpina

(Caryophyllaceae) populations on mining sites. Folia Geobot v. 34, p: 471-481. 1999. https://doi.org/10.1007/BF02914923

PECKOLT, T., PECKOLT, G. História das plantas medicinais e úteis do Brasil. Belo Horizonte: Fino Traço. 2016. $904 \mathrm{p}$.

POSEY, D. Manejo de floresta secundária, capoeira, campos e cerrados (Kayapó). Ribeiro, D., Ribeiro, B. G.

(coord.). Suma Etnológica Brasileira. Vol. 1: Etnobiologia. Petrópolis: Vozes, Finep. 1986. p. 172-186.

PRIMAVESI, A. M. Algumas plantas indicadoras - como reconhecer os problemas do solo. São Paulo:

Expressão Popular. 2017. 48p.

SAINT-HILAIRE, A. Flora Brasiliae Meridional. v.1. Paris. 1825.

SILVA JUNIOR, M. C. 100 arvores do Cerrado: guia de campo. Brasília: Editora Rede de Sementes do

Cerrado. 2005. 278 pg

SILVEIRA, F. V., NETO, I.C., CUNHA, L.M. Projeto Diamante Brasil. In 7th Simpósio Brasileiro de

Geologia do Diamante, Salvador. 2018. Disponível em file:///C:/Users/btomc/Downloads/Silveiraetal.2018b.pdf SPIX, J. B., MARTIUS, C. F. Travels in Brazil in the years 1817-1820. Undertaken by command of His

Majesty the King of Bavaria, Longmans, London. 1824.

SVISERO, D. P., CHIEREGATI, L. A. Contexto geológico de kimberlitos, lamproítos e ocorrências

diamantíferas do Brasil. Boletim IG-USP 9. 1991.

TROPICOS. Missouri Botanical Garden. 2020. Disponível em: https://www.tropicos.org/home, (acesso em 01/02/2020).

VALE, E. Perfil da exploração de diamantes no Brasil: relatório final. Brasília: SMM/MME. 2003. 69p. 